\title{
Approche quantitative des interactions soignantes à l'hôpital psychiatrique depuis une perspective interactionniste
}

\section{A quantitative approach to caregiver interactions in the psychiatric hospital from an interactionist perspective}

\author{
Benoit Chalancon ${ }^{1}$, Isabel Colón de Carvajal ${ }^{2}$, Louis Maritaud ${ }^{2}$, Justine Lascar ${ }^{2}$ et Laurie \\ Boyer $^{2}$ \\ 1 Centre hospitalier le Vinatier, France \\ 2 Laboratoire ICAR UMR 5191 (CNRS, ENS de Lyon, Université Lumière Lyon 2), France
}

\begin{abstract}
Résumé. La réforme du système de santé incite les soignants à être de plus en plus efficaces lors de situations de communication. Les spécificités de la pratique infirmière en santé mentale peuvent s'illustrer par le soin relationnel qui représente un enjeu dans la continuité des soins au vu de la complexité grandissante des prises en charge. Dans un tel contexte, une étude portant sur les compétences d'interaction des infirmiers dans un service psychiatrique pour adultes semble se justifier. L'ancrage théorique et méthodologique de cette recherche relèvent de l'analyse conversationnelle et de l'ethnométhodologie. Le projet CIPSY est ainsi un projet exploratoire qui étudie les compétences d'interaction durant les relèves infirmières, et les multiples façons de transmettre de l'information disponibles dans leur pratique, qu'elles soient écrites ou orales. Cet article présente d'une part la méthode exploratoire utilisée et propose une analyse quantitative du temps passé dans chaque relève enregistrée, comparant en particulier les patients en mesure d'isolement et les patients en chambre simple. D'autre part, nous présentons trois résultats statistiques soulignant les différences de contenu des relèves orales et écrites. Nous décrivons enfin l'ébauche d'une méthode utilisée pour visualiser les données orales d'un même patient à l'aide du logiciel Voyant Tools.
\end{abstract}

\begin{abstract}
The reform of the health system organization encourages medical teams to be efficient when dealing with communication issues. Considering the specificities involved in nursing practice in mental health through relational care, the continuity of care and the growing complexity related to care, there is an increasing number of caregivers dealing with patients. In such context, a study focusing on the interaction skills of current nurses based in the psychiatry service for adults seems to be relevant. The design of fieldwork is the one developed in ethnomethodology and conversation analysis. Therefore, the CIPSY project is an exploratory project aiming to study the interaction skills of nurses during handovers, and the multiple ways to transmit information available in their practice, whether orally or written. This paper first presents the exploratory method used and proposes a quantitative analysis of the time spent in each recorded handover, comparing the time spent on the usual patients in the structure and the ones isolated from the others. Then we present three statistical results highlighting the differences in the content of the oral and written readings and we describe a draft method used to visualize oral data from a single patient using Voyant Tools software.
\end{abstract}




\section{Introduction}

Les échanges verbaux en contexte de relève infirmière ont fait l'objet de différentes études depuis plusieurs années, dans des contextes différents (service de chirurgie, de pédiatrie, d'urgence, de psychiatrie, etc.). Dans le domaine des soins infirmiers en santé mentale, plusieurs études se sont intéressées aux pratiques des relèves. À l'international, Cleary, Walter et Horsfall (2009) ont interrogé par exemple la nécessité d'un changement dans les pratiques de fins d'hospitalisation. Quelques années plus tard, une équipe de recherche australienne (Cowan et al., 2018) a travaillé à l'élaboration de quelques lignes directrices pour un contenu et un processus structuré dans la relève infirmière en santé mentale. Nous pouvons citer également les travaux sur la coopération entre infirmiers et médecins (Lanza et al., 2004) ou sur le discours rapporté dans des récits pendant la relève (Bangerter, Mayor et Pekarek Doehler, 2011). Plus récemment, Abraham et al. (2015) ont mené une analyse interactionnelle et séquentielle de la structure et des contenus d'une relève; LeBaron, Christianson et Garrett (2016) ont quant à eux conduit une étude ethnométhodologique des routines conversationnelles ; enfin Mori, Imamura et Shima (2017) ont montré comment les infirmières travaillant dans un service gériatrique japonais rapportent, confirment et reconstruisent les informations lors des relèves. En France, Grosjean (1997, 2004) et Grosjean \& Lacoste (1999) se sont particulièrement intéressées à décrire les pratiques des professionnels dans ce contexte. L'édition de 1999 constitue un ouvrage de référence sur le sujet des relèves. Il apparaît donc que l'étude des relèves infirmières dans le contexte spécifique de la psychiatrie et de la santé mentale n'a semble-t-il pas été développée dans une approche interactionnelle.

La situation actuelle de la santé mentale augmente de plus en plus le nombre d'intervenants autour du patient, le développement des spécialisations en psychiatrie multipliant les acteurs à son contact. Ce paysage, où les collaborateurs se diversifient au service du soin, met également en avant toute l'importance d'une communication interprofessionnelle performante. Cette complexification favorable aux soins donne une dimension toute particulière aux transmissions orales et écrites dans les équipes soignantes.

Nous faisons l'hypothèse qu'il existe des différences de contenus entre les relèves infirmières orales et écrites. Nos analyses visent à objectiver un éventuel décalage entre les écrits infirmiers et les échanges oraux des relèves, par le biais d'indicateurs linguistiques (syntaxiques, lexicaux, interactionnels, etc.), observés dans les cadres théoriques et suivant les méthodologies spécifiques en analyse de productions textuelles et en analyse du discours.

Pour y répondre, nous posons trois hypothèses :

1. Les stratégies d'expressions sont différentes à l'oral et à l'écrit et s'expriment par des indicateurs spécifiques selon la modalité (il peut exister des différences dans la fréquence et l'apparition ou la distribution et la topologie de ces indicateurs).

2. Ces spécificités caractérisent une ou des fonctions propres à chaque type de relève, selon le temps, selon le destinataire (fonction de recherche d'action immédiate tournée vers le futur proche, fonction de protection dite " parapluie » tournée vers le passé).

3. Certains thèmes abordés à l'écrit ou à l'oral sont plus difficiles à exprimer dans l'autre modalité de transmission (législatif, contrainte, sexualité, etc.).

Après avoir présenté la méthode appliquée pour l'accès au terrain et la captation des données, nous proposons une analyse quantitative du temps passé dans chaque relève enregistrée, en comparant la durée moyenne d'une relève pour les patients en isolement d'une part, et pour les autres patients d'autre part. Puis, nous expliquons le travail réalisé pour la transcription et le codage des données orales à l'aide du logiciel ELAN ; et nous présentons le codage des données écrites avec le logiciel CLAN. À partir de ce travail de codage (environ $450 \mathrm{~h}$ ), nous 
présentons un second travail comparatif, sur les données orales et écrites de sept patients qui ont été transcrites et codées. Enfin, nous décrivons l'ébauche d'une méthode utilisée pour investiguer et visualiser les données orales d'un même patient à l'aide du logiciel Voyant Tools.

\section{Travail de terrain et captation des données}

Le projet CIPSY est une étude observationnelle, constituée d'un corpus de données réalisé au sein d'un service de psychiatrie adulte. L'analyse se déploie d'un point de vue à la fois quantitatif et qualitatif ${ }^{1}$. La méthodologie de travail de terrain, de recueil de corpus et d'analyse dans laquelle nous nous inscrivons est celle développée en ethnométhodologie et en analyse conversationnelle. Cette méthodologie vise à constituer un corpus de données audio et vidéo afin de rendre disponibles, et donc analysables, les détails linguistiques, multimodaux et situationnels (regards, gestes, mouvements, actions, objets, cadre physique) pertinents pour l'interaction enregistrée (Groupe ICOR, 2006). Elle permet aussi d'observer précisément l'évolution des interactions sociales, ou plutôt soignantes dans notre cas, qui se co-construisent entre les participants, dans le temps et dans l'espace de l'activité.

Le choix du lieu de tournage a en premier lieu suivi tout un parcours d'autorisations institutionnelles au sein de l'hôpital. L'étude est alors perçue comme une opportunité pour l'encadrement des équipes. L'une d'entre elles a été sollicitée pour la réalisation des enregistrements. Après la présentation de l'étude aux personnels de jour et de nuit ainsi que le recueil des autorisations de droit à l'image, nous avons programmé les jours de tournage dans le courant de l'été 2018. L'équipe filmée a choisi d'enregistrer les lundis uniquement. En effet, le lundi, premier jour de la semaine, est d'une richesse particulière pour un service de psychiatrie adulte puisque abondant d'évènements cliniques du week-end. La particularité de ce jour tient également au déroulement du staff hebdomadaire, qui est une réunion pluriprofessionnelle regroupant des soignants médicaux (médecins, psychiatres, etc.) et nonmédicaux (infirmiers, aide-soignants, etc.). Ce corpus audiovisuel original, d'une durée totale de 14 heures 30, met en situation 38 professionnels de santé, et il est fait mention de 40 patients différents lors de ces relèves (sur cinq lundis consécutifs, à raison de 4 relèves par jour à $6 \mathrm{~h}, 9 \mathrm{~h}, 14 \mathrm{~h}$ et $21 \mathrm{~h} 30$ ).

Les relèves de postes inter-équipes ont été enregistrées par un dispositif audiovisuel composé de trois caméras et cinq micros. À des fins de discrétion, nous avons utilisé des caméscopes sur trépied et une action cam. La prise de son est assurée par des micro-cravates ainsi qu'un micro d'ambiance déposés sur les tables. L'équipe de recherche est présente uniquement au moment d'allumer et d'éteindre le dispositif, limitant ainsi le contact avec l'équipe de soin. En parallèle de ces actions, les données écrites dans les dossiers de soins informatisés ont été également collectées et correspondent aux cinq lundis enregistrés.

Après la phase d'enregistrement des données orales et du recueil des données écrites, l'équipe de recherche du projet a réalisé une synchronisation des différentes pistes audio et vidéo (voir Image 1). Le corpus comprend 60 sources vidéo et 80 sources audio différentes, composées en 20 vues synchronisées.

\footnotetext{
${ }^{1}$ Les auteurs déclarent avoir reçu un soutien financier pour la réalisation de cette étude de la part du LABEX ASLAN (ANR-10-LABX-0081) de l'Université de Lyon dans le cadre du programme «Investissements d'Avenir» (ANR-11-IDEX-0007) de l'État Français géré par l'Agence Nationale de la Recherche (ANR) ; mais également de la part du Comité Scientifique de la Recherche et l'Université Lyon 2 - Centre Hospitalier le Vinatier. Les auteurs remercient le laboratoire ICAR (UMR 5191), le LABEX ASLAN (ANR-10-LABX-0081) ainsi que le Comité Scientifique de la Recherche et l'Université Lyon 2 - Centre Hospitalier le Vinatier pour leur soutien.
} 


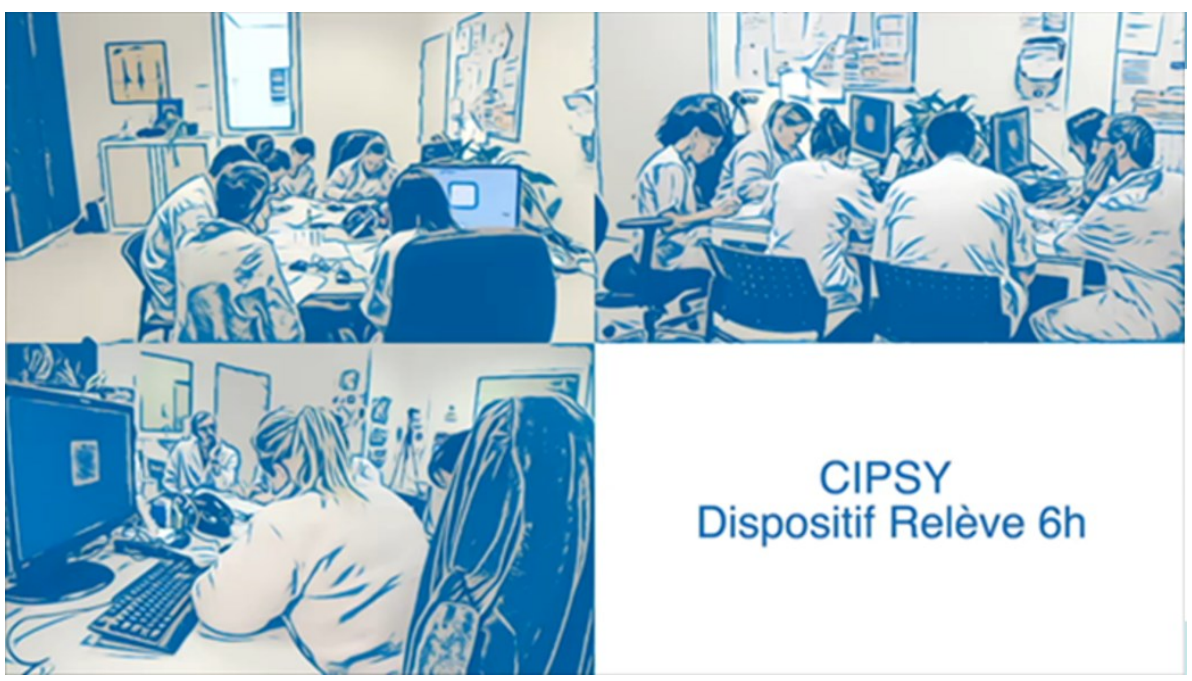

Image 1. Montage multiscope synchronisé des relèves orales ( 3 sources vidéo +4 sources audio) anonymisées à l'aide d'un filtre Cartoon sur Final Cut Pro

\section{Analyse statistique générale et descriptive des relèves de soin}

Dans l'objectif de réaliser une analyse descriptive des données, nous avons constitué un fichier Excel sur lequel est reporté, à la seconde près, les durées des échanges pour chaque patient mentionné durant les relèves. La première colonne représente la liste des pseudos des patients, puis la première ligne indique le temps de début et le temps de fin de chaque mention du patient en fonction du jour et de la relève concernée. Chaque saisie initiale du temps de début et de fin a été vérifiée par une deuxième personne de l'équipe afin de limiter les erreurs d'appréciation. À partir de ce tableau, nous avons élaboré des statistiques sur les durées des relèves variables dans la journée du lundi, en comparant également le temps de parole dédié pour certains profils de patient par rapport à d'autres. Nous présentons maintenant ces premiers résultats.

\subsection{Durée moyenne journalière des relèves}

La relève de 6 heures, qui se déroule dans le bureau infirmier, réunit les infirmiers, le plus souvent deux professionnels de jour, et un de nuit. Ils réalisent la première transmission orale de la journée, pendant que les aides-soignants (un de jour et un de nuit) réalisent un tour physique du service. La durée moyenne de la relève du matin est de 17 minutes, et varie seulement de 3 minutes entre les différents jours de tournage. Cette régularité notable s'explique en partie par la présence d'une même professionnelle lors de quatre des cinq transmissions matinales étudiées.

Le temps des transmissions orales de 14 heures, croise les équipes du matin et de l'après-midi au complet, réunissant entre 8 et 12 personnes. Il a lieu dans la salle de réunion pour pouvoir d'une part accueillir toute l'équipe mais également pour l'utilisation du vidéo projecteur. Ce temps présente la plus grande variabilité observée sur nos cinq jours de tournage, puisque les durées peuvent doubler d'un jour à l'autre. La moyenne est de 44 minutes, et notons que cette durée dépasse de 14 minutes le temps initialement prévu par l'organisation des soins.

La relève de 21 heures 30 croise l'équipe de l'après-midi et l'équipe de nuit. Elle ressemble en termes d'organisation et de durée à la première transmission de la journée à 6 heures sans 
toutefois avoir la même régularité puisque les durées varient du simple au double, pour une moyenne de 31 minutes.

Enfin, la relève de 9 heures dite "réunion médicale de la semaine », a lieu dans le même contexte que celle de 14 heures, c'est-à-dire dans la salle de réunion, avec le vidéo projecteur. L'objectif de cette relève est différent, il s'agit d'interroger en équipe le projet de soin du patient, et l'évolution de son état de santé. C'est un temps de co-construction pluriprofessionnelle au service des patients. Ces durées d'échanges ont un delta qui varie de 19,4 $\%$ pour une durée maximale observée de 93 minutes (voir figure 1).

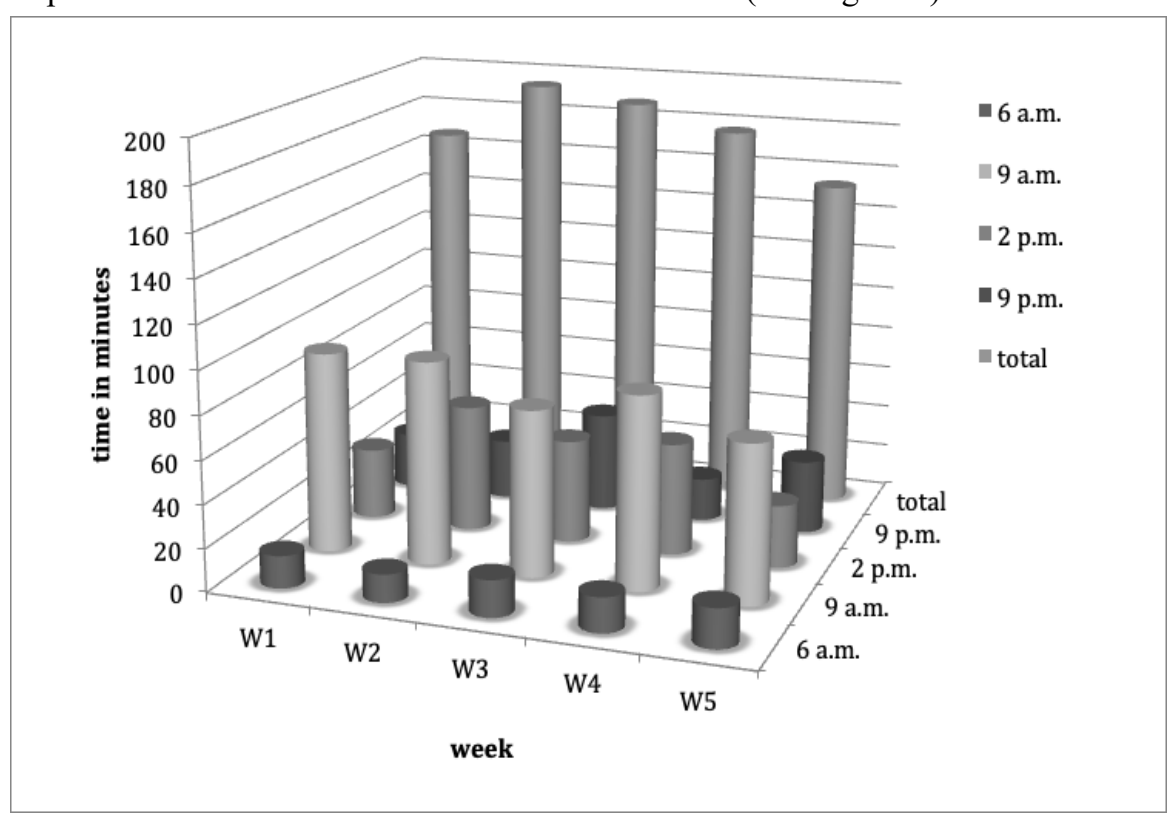

Figure 1. Durée en minute par relève et par semaine

\subsection{Moyenne des temps d'échanges du patient en chambre simple ou en chambre d'isolement}

Dans un second temps, nous avons mesuré les moyennes de temps d'échanges des patients installés en chambre simple, puis nous les avons comparés aux temps d'échanges moyens pour les patients en chambre d'isolement. Cet endroit est également nommé ESPI pour Espace de Soin Psychiatrique Intensif.

Ainsi pour la relève de 6 heures, la durée moyenne par patient en chambre simple est de 30 secondes, c'est la plus courte sur les quatre temps d'échanges. La durée moyenne pour un patient en isolement est de 3 minutes, autant qu'à la relève de 21 heures 30 .

Le staff médical de 9 heures offre le temps d'échanges le plus conséquent au patient en isolement, avec 10 minutes en moyenne contre 3 minutes pour les autres patients en chambre simple. Notons que la relève de 14 heures offre également un temps d'échanges conséquent au patient en ESPI, puisqu'il occupe en moyenne 7 minutes le débat, pour une durée totale de 45 minutes.

L'impact sur les durées (exprimées en moyenne) est indiqué en pourcentage. Nous observons ainsi qu'en termes de proportion le temps de relève concernant les patients en isolement occupe $18 \%$ du temps à la relève de 6 heures, montrant une préoccupation des équipes de nuit à transmettre les informations du patient en isolement. Les proportions de 9 heures et 21 
heures, respectivement $12 \%$ et $10 \%$, sont plus lissées et montrent un intérêt plus généraliste pour l'ensemble des patients de l'unité dans un moment où le nombre de professionnels est le plus important.

En conclusion, et comme nous pouvions nous y attendre, les patients en situation d'isolement occupent une place prioritaire dans les relèves et réunions pluridisciplinaires en service de psychiatrie adulte.

\section{Transcription et codage des données orales et écrites}

En parallèle des analyses quantitatives sur la durée des relèves enregistrées sur les cinq lundis consécutifs, nous avons réalisé deux étapes permettant d'analyser les contenus interactionnels produits par les soignants à l'oral en comparaison avec les propos écrits dans le dossier des patients : il s'agit, d'une part, de la transcription d'une partie des données orales et de l'adaptation des dossiers patients sauvegardés dans un format exploitable ; et d'autre part, du codage de catégories d'analyses observables (à des échelles de granularité très variées) sur la base d'une grille d'annotation construite en équipe en tenant compte des objectifs donnés dans le projet CIPSY. Pour ce double travail, nous avons utilisé deux logiciels spécifiques d'annotation :

a) $\mathrm{ELAN}^{2}$, pour les données orales, permet, en particulier, la transcription $\mathrm{du}$ discours, des gestes, des regards produits par les participants pendant les relèves ainsi que l'annotation multi-niveau (syntaxique, lexical, référentiel, pragmatique, etc.) de catégorie d'analyses définies en amont le plus souvent à des fins statistiques ;

b) $\mathrm{CLAN}^{3}$, pour les données écrites, permet d'annoter le discours au moyen de requêtes spécifiques selon des mots clés ou des marqueurs syntaxiques.

Nous allons ainsi expliquer plus en détail la méthodologie appliquée aux données orales et écrites car elle constitue, pour l'équipe de recherche, un long travail de réflexion et de coconstruction (y compris avec l'équipe de soin) sur plus de deux ans, afin de répondre au mieux aux objectifs du projet tout en tenant compte du coût financier et humain que ce travail implique. Nous pensons que ce partage des pratiques peut servir d'autres collègues qui souhaiteraient utiliser les outils tels que ELAN et CLAN à des fins d'analyses qualitatives et quantitatives.

\subsection{Utilisation d'ELAN pour l'analyse des données orales}

Sur les 14 heures 30 de données totales, nous avons pu transcrire à ce jour la totalité des relèves concernant sept patients, ce qui représente $25,51 \%$ du corpus, soit 3 heures 20 minutes de données orales. Le choix de ces sept patients s'est porté sur leur modalité de séjour hospitalier.

- patient 1 cesel : il a débuté son séjour durant l'étude, et a été placé dans l'espace de soin psychiatrique intensif.

- patient 2 dubyv : il est sorti, puis a été ré-admis.

- patient 3 lebba : il a terminé son séjour durant le tournage.

\footnotetext{
${ }^{2}$ ELAN Linguistic Annotator, (C Max Planck Institute for Psycholinguistics, https://tla.mpi.nl/tools/tlatools/elan/

3 Computerized Language Analysis, (C) Leonid Spektor at Carnegie Mellon University, https://talkbank.org/
} 
- patient 4 legco : il a commencé son séjour hospitalier.

- patient 5 borma $:$ il est sorti après la première semaine de tournage

- patients 6 touje et 7 delem : ils ne sont ni rentrés, ni sortis durant la phase de captation, étant ainsi au cœur de leur hospitalisation.

La transcription (uniquement verbale) de cet échantillon a nécessité 250 heures de travail ${ }^{4}$.

L'analyse des données s'appuie également sur un long travail d'annotation / de codage (300 heures $^{5}$ ) rendu possible grâce à l'élaboration d'une grille de codage initiée par l'équipe de recherche, puis confrontée et enrichie auprès d'un panel d'experts, témoignant d'une expérience notable dans l'exercice en soin. Cette même grille a été également présentée et enrichie auprès de l'équipe de soin partenaire au projet. Elle est commune pour l'annotation des données orales et écrites, afin de favoriser la comparaison entre les deux modalités de partage d'informations.

La grille de codage comprend 16 lignes d'observables (qu'on appelle "acteur" ou "tiers" dans le logiciel) ${ }^{6}$.

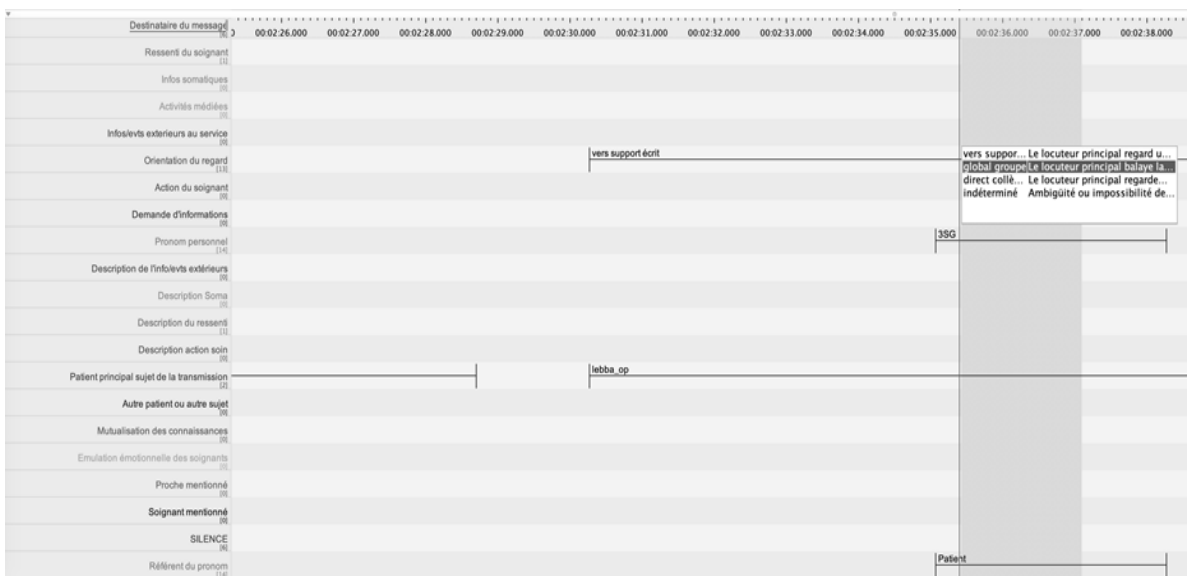

Image 2. Capture d'écran des lignes de codage dans ELAN

\subsection{Utilisation de CLAN pour les données écrites}

Pour les 300 notes écrites et transcrites pour l'ensemble des patients, environ 150 heures de travail ont été nécessaires. À partir de l'extraction des observations écrites fournie par le département d'informations médicales (DIM) du Vinatier sous forme d'un tableur Excel, nous avons construit des fichiers séparés pour chaque relève et chaque patient, en respectant les normes du logiciel. Nous avons donc décidé, pour coller au mieux aux données, de séquencer les productions écrites en clauses, et de remplacer les éléments de ponctuation par des codes que CLAN ne prenait pas en compte lors de l'analyse des données. Pour ce faire, nous avons établi une nomenclature de fichiers comprenant le nom du patient, l'heure et la semaine de saisie de l'observation, ainsi que le type d'observation et la fonction du scripteur, sous ce format : «CIPSY_S2_05h48_Cesel_OAS_AS », avec «OAS » correspondant à « observation aide-soignant » et « $\mathrm{AS}$ » à aide-soignant.

\footnotetext{
${ }^{4}$ Nous remercions Laurie Boyer, Lydia Heiden et Louis Maritaud pour ce travail.

${ }^{5}$ Nous remercions Lydia Heiden, Elizaveta Chernyshova, Louis Maritaud et Benoît Chalancon pour ce travail.

${ }^{6}$ http://icar.cnrs.fr/cipsy/corpus/
} 
Une fois cette mise en forme faite pour être compréhensible par le logiciel, nous avons adapté la grille de codage orale, et l'avons rendue semi-automatisable dans son utilisation dans CLAN, en créant un fichier «.cut». Par la suite, nous avons codé les données écrites et mené les analyses.

\subsection{Première étude comparative sur sept patients}

Nous avons réalisé une première étude comparative entre les données orales et les données écrites sur les sept patients transcrits et annotés à partir de notre grille de codage. Cette étude permet de rendre compte de premiers résultats quantitatifs pour mieux comprendre les informations communes et différentes, partagées à la fois à l'oral et à l'écrit pour ces sept patients, mais également de relever certaines différences où des informations vont être majoritairement communiquées à l'oral alors qu'elles seront absentes à l'écrit.

Pour cet article, nous avons retenu et comparé trois observables : 1) les actions mentionnées par les soignants pendant leur relève ; 2) les proches des patients mentionnés ; 3) les pronoms personnels employés par les soignants.

Pour rappel, les sept patients dont il est question dans cette première étude quantitative sont restés un nombre de semaines différent, et n'ont pas fait l'objet de transmission sur le même nombre de relèves. Ainsi, Delem et Touje sont restés cinq semaines et ont été mentionnés dans 19 relèves ; Dubyv et Legco sont restés trois semaines et ont été mentionnés dans 12 relèves ; Lebba et Cesel sont restés trois semaines et ont été mentionnés dans 11 relèves. Enfin, Borma n'est resté qu'une semaine et a été mentionné dans 4 relèves seulement.

\subsubsection{Actions mentionnées par les soignants}

Dans cette catégorie, nous avons codé les actions des soignants mentionnées pendant la relève. Nous avons distingué le fait que ces actions pouvaient être soit mentionnées et réalisées (donc axées sur un état passé), soit mentionnées mais non réalisées (donc axées sur un état futur). Les deux graphiques suivants montrent la répartition des mentions d'action des sept patients, d'une part dans les relèves orales (voir figure 2 ) ; et d'autre part, dans les dossiers écrits (voir figure 3).

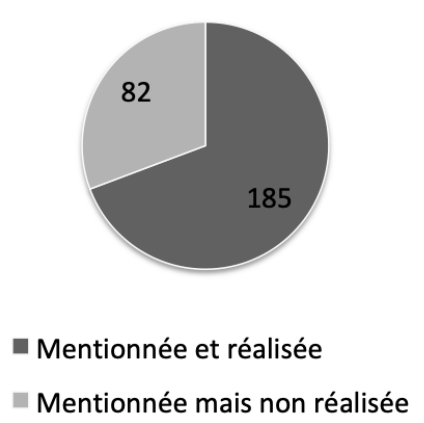

Figure 2. Actions des soignants à l'oral

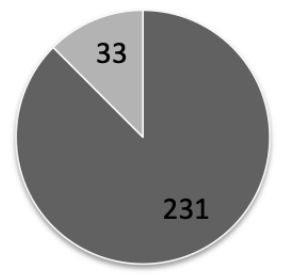

- Mentionnée et réalisée

Mentionnée mais non réalisée

Que ce soit à l'écrit ou à l'oral, les actions des soignants sont majoritairement mentionnées et réalisées. Les soignants partagent ainsi, aussi bien à l'écrit qu'à l'oral, les actions passées. Aussi, la proportion de mentions d'actions réalisées est nettement plus importante à l'écrit (248 contre 185 à l'oral). Cette proportion plus significative à l'écrit peut s'expliquer par le besoin de laisser plus de traces écrites dans le dossier patient sur les actions réalisées afin de 
garantir une traçabilité des soins prodigués sur les patients.

Concernant les actions mentionnées mais non réalisées (donc plutôt projetées vers une réalisation à venir), on remarque une verbalisation de ces actions beaucoup plus présentes à l'oral, mais les soignants laissent moins de traces écrites de ces soins à venir dans les dossiers écrits des patients. Cette différence peut s'expliquer par le fait que les soignants peuvent parfois ajuster les traitements des patients au fil des heures, en fonction de leur état. Les mentions de ces actions à venir dans le dossier patient sont donc moins obligatoires ou indispensables. Elles ont du sens au fil des heures de la journée ou de la nuit, pendant la pratique des soignants, mais n'ont pas lieu d'être dans le dossier écrit. Il est possible aussi qu'une action mentionnée mais à réaliser (future) pendant la transmission orale deviennent finalement une action mentionnée et réalisée (passée) dans le dossier écrit, ce qui explique également cette diminution à l'écrit, mais l'augmentation des actions réalisées à l'écrit.

\subsubsection{Proche mentionné}

La deuxième catégorie d'information analysée concerne les moments où les soignants ont fait mention des proches des patients. Ainsi, nous avons au total 99 mentions de proche sur les sept patients étudiés, dans les relèves orales. Le graphique suivant (voir figure 4) montre par ordre décroissant la fréquence des ces mentions en fonction du type de proche.

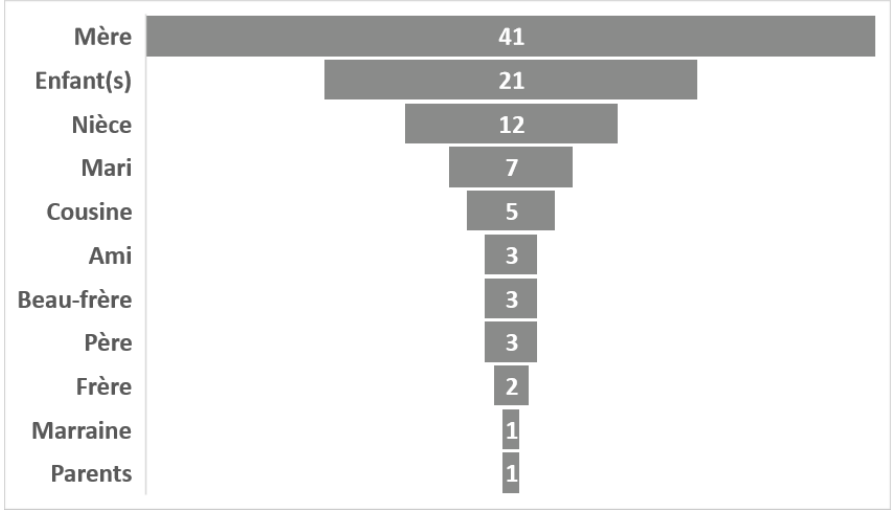

Figure 4. Répartition en fonction des proches mentionnés par les soignants dans les relèves orales

On remarque ainsi que la "mère » des patients est le proche le plus mentionné par les soignants. Il s'agit d'un proche pour cinq des sept patients. Si l'on regarde plus en détail, il y a certains patients pour qui il est fait mention d'une variété de proches alors que pour d'autres, il ne s'agit que d'un seul type de proche. Ainsi, quand les soignants parlent de Delem (qui est restée cinq semaines), ils évoquent jusqu'à six types de proches dans son entourage ; alors que lorsqu'ils parlent de Legco ou Lebba, il n'est fait mention que d'un seul proche (qui sont tout de même restés trois semaines chacun).

Si l'on compare à présent avec le nombre de mentions de proche à l'écrit, on observe un écart très important puisque nous relevons seulement 11 mentions de proche dans quatre des sept dossiers patients (4 pour Legco et Cesel, 2 pour Delem et 1 pour Touje). Si cet écart ne trouve aujourd'hui pas d'explications, il est certain que maintenir et préserver les relèves orales, permettra de mieux comprendre les dynamiques des entourages familiaux et amicales des personnes concernées. Lors des transmissions orales, les soignants donnent donc une place importante à l'évocation de ces proches pour partager des récits de vie des patients dans leur cadre familial. La transmission orale serait donc un lieu d'échange privilégié pour ces moments de récits de vie concernant l'entourage du patient ; à la différence du dossier écrit 
dans lequel quelques soignants relatent ponctuellement un proche d'un patient qui a pu avoir un rôle particulier ce jour-là pour le patient. Enfin, à l'écrit, l'information est beaucoup plus ancrée dans le présent par rapport à l'oral où les soignants peuvent évoquer plus facilement et en détail les événements passés des patients, relatifs à leurs proches.

\subsubsection{Pronom personnel}

La troisième et dernière catégorie étudiée concerne l'emploi des pronoms personnels dans les relèves orales et écrites. Cette catégorie nous semble particulièrement intéressante pour comprendre la diversité des personnes impliquées dans l'accompagnement des patients. À l'annotation des pronoms personnels, nous avons en effet associé l'annotation des référents à ces pronoms. Ces référents peuvent être le patient, l'équipe, la sous-équipe ou le soignant. Mais parfois de façon plus complexe, ces référents peuvent renvoyer à quelqu'un d'autre, ou à un référent impersonnel.

Les graphiques ci-après illustrent la répartition d'emploi des pronoms lors des relèves orales (voir figure 5) et celle lors des relèves écrites (voir figure 6).

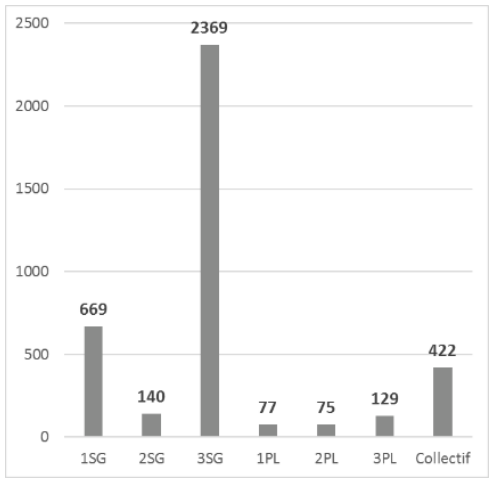

Figure 5. Pronoms personnels à l'oral

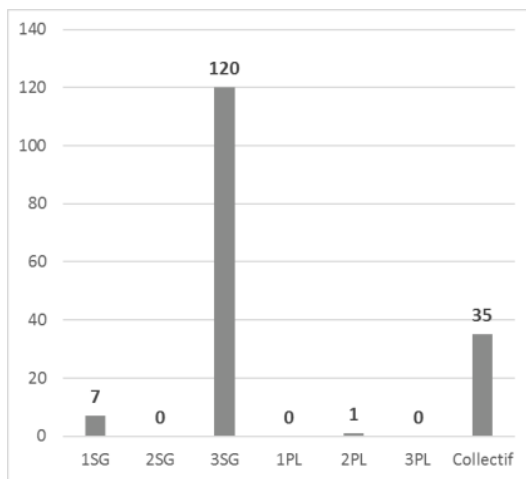

Figure 6. Pronoms personnels à l'écrit

On remarque clairement une large majorité d'emploi de pronoms à la troisième personne du singulier, aussi bien à l'oral qu'à l'écrit, avec une quantité d'occurrence importante particulièrement à l'oral. Cela s'explique par le fait que lors des transmissions orales, les soignants font souvent référence au patient lors de discours rapporté en parlant du patient à la troisième personne du singulier. C'est l'activité même de transmission orale qui construit un espace d'énonciation à la troisième personne du singulier.

En comparant les données à l'oral et à l'écrit, on note également que l'ensemble des pronoms sont employés à l'oral, alors qu'à l'écrit le «tu», « nous » et « ils » ne sont jamais utilisés. Là encore, l'activité même de transmission orale construit un cadre d'énonciation multiparticipant qui permet un usage complet des pronoms personnels, alors qu'à l'écrit, les soignants font mention d'informations en leur nom (« je »), en évoquant le ou la patiente (« il, elle ») ou le collectif (l'équipe ou la sous équipe par exemple).

\section{Exploration du lexique "traitement/prescription » sur un patient via Clan et Voyant Tools}

Pour pouvoir utiliser des logiciels orientés vers de la textométrie sur nos transcriptions orales, un travail d'adaptation des conventions a été opéré. En effet, puisque nous avons en premier lieu travaillé à partir des conventions ICOR (2013), une série de caractères, utiles dans une 
démarche interactionnelle fine, rendait la reconstruction des analyses globales trop lourdes. Ainsi, nous avons opté pour des scripts en langage Python ${ }^{7}$ afin de supprimer les différentes annotations propres aux conventions ICOR et rendre les fichiers compréhensibles par des outils de textométrie c'est-à-dire de revenir à une transcription orthographique standard de nos données. Nous avons conçu 3 scripts différents, selon deux paramètres : le logiciel visé, et l'orientation des analyses à mener.

Dans un premier temps, notre choix s'est porté sur Voyant Tools. Nous avons également ajouté dans les scripts la possibilité de créer des collections à partir du corpus transcrit selon les orientations différentes des analyses à mener. En effet, puisque l'intérêt pouvait porter à la fois sur les productions des différents locuteurs à différentes relèves, mais aussi sur le contexte d'apparition des divers mots ou syntagmes (c'est-à-dire des groupes de mots fonctionnant comme un ensemble, comme par exemple les groupes nominaux), deux types d'outputs (par output on désigne ce que produit le script lors de son exécution) ont été conçus. Le premier réunit l'intégralité des productions d'un participant à une relève, chaque tour de parole étant alors séparé par l'usage d'un point («.»). Cette première collection nous a permis de voir le lexique différent utilisé pour chaque patient, l'évolution au fil des relèves du nombre de mots utilisés par patient.

Le deuxième type d'output est quant à lui constitué des différentes prises de paroles de tous les locuteurs, dont l'ordre séquentiel est conservé, mais en retirant les pseudos pour qu'ils ne soient pas pris en compte dans les fréquences de mots. À partir de ces premiers essais sur Voyant Tools, il nous est apparu comme pertinent de se concentrer sur plusieurs déterminants : les champs lexicaux employés par les soignants selon leur profil et l'heure de relève, le recours à des tournures exprimant des opinions personnelles (telles que « je crois » ou « je pense »), ainsi que le contexte d'apparition des différents tokens étudiés. Afin de sélectionner ces éléments, nous avons procédé de façon empirique. Voyant Tools nous a permis de faire des repérages de segments saillants grâce aux visualisations automatiques. Ensuite, à partir de ces sélections, notre intérêt s'est porté vers le logiciel CLAN, pour procéder à une lemmatisation des transcriptions, et utiliser la commande freq pour pouvoir dégager des tendances.

CLAN est un logiciel qui accepte des transcriptions suivant une certaine forme. Il lui est nécessaire d'avoir des lignes d'en-tête spécifiques, des pseudos de locuteurs associés aux tours de parole ainsi que des points à la fin de chaque production associée à un participant. Nous avons donc repris le script d'allègement des conventions ICOR, et l'avons adapté au format CLAN.

La solution du parsing présente plusieurs intérêts : elle est reproductible et pourra être utile dans des analyses futures. D'autre part, les scripts sont adaptables, et la démarche peut être dupliquée dans d'autres projets, l'outil est relativement simple en termes de conception et d'utilisation. De plus, puisque le traitement des données est automatique, il s'agit d'un gain de temps important dans les manipulations des conventions.

Dans notre cas, nous avons pu mettre en évidence une utilisation particulière du champ lexical du traitement d'un patient en chambre d'isolement (en l'occurrence, Cesel). En définissant une série de termes d'un même champ lexical, puis en menant une analyse de fréquence sous CLAN, nous avons pu constater par exemple que contrairement à ce que nous pourrions croire, le traitement de ce patient est plus discuté lors de la relève de 14 heures que durant le staff hebdomadaire de 9 heures :

${ }^{7}$ Créé par Guido Von Rossum et développé par la Python Software Foundation, il s'agit d'un langage informatique très utilisé permettant entre autres l'automatisation de certaines tâches par le biais de scripts. Les auteurs remercient, à ce propos, Louise Tarrade, qui a été d'une grande aide dans la conception de ces scripts. 
Tableau 1. Nombre de tokens relatifs au traitement par heure de relève

\begin{tabular}{|c|c|c|}
\hline Heure de réunion & Nombre de tokens & Ratio \\
\hline $\mathbf{6}$ heures & 16 & $23,19 \%$ \\
\hline $\mathbf{9}$ heures & 17 & $24,64 \%$ \\
\hline $\mathbf{1 4}$ heures & 24 & $34,78 \%$ \\
\hline $\mathbf{2 1}$ heures & 12 & $17,39 \%$ \\
\hline
\end{tabular}

De la même manière, nous avons pu considérer le nombre de tokens énoncés par les différents profils de soignants :

Tableau 2. Répartition des tokens en lien avec le traitement selon la profession du locuteur

\begin{tabular}{|c|c|c|c|c|}
\hline $\begin{array}{c}\text { Fonctions des } \\
\text { locuteurs }\end{array}$ & $\begin{array}{c}\text { Nombre de } \\
\text { locuteurs }\end{array}$ & $\begin{array}{c}\text { Nombre de } \\
\text { tokens }\end{array}$ & Ratio & $\begin{array}{c}\text { Nombre de } \\
\text { token moyen } \\
\text { par } \\
\text { professionnel }\end{array}$ \\
\hline Infirmier & 37 & 63 & $91,30 \%$ & 1,7 \\
\hline Psychiatre & 2 & 2 & $2,90 \%$ & 1 \\
\hline Aide-Soignant & 3 & 2 & $2,90 \%$ & 0,6 \\
\hline Interne & 2 & 2 & $2,90 \%$ & 1 \\
\hline
\end{tabular}

Ces deux derniers tableaux nous indiquent que le statut des soignants n'est pas nécessairement directement lié à leur utilisation de tokens propres au traitement. En effet, les infirmiers n'ont pas de pouvoir de prescription en tant que tel, mais leur champ d'action en terme de dispensation et de surveillance des traitements les amènes régulièrement dans le champ lexical lié aux médicaments. Or, puisque la réunion lors de laquelle les traitements sont le plus discutés est une relève infirmière, sans soignants médicaux, et que le nombre moyen de tokens ayant un rapport au traitement est supérieur chez les infirmiers que chez les autres professions représentées, nous pouvons constater qu'une part active des échanges au niveau des traitements est prise en charge par ce corps de métier.

Enfin, nous avons cherché à voir si une évolution était notable selon les semaines de production des tokens : 
Tableau 3. Répartition des tokens par semaine d'enregistrement

\begin{tabular}{|c|c|c|}
\hline Semaine & Nombre de tokens & Ratio \\
\hline S2 & 1 & $1,45 \%$ \\
\hline S3 & 18 & $26,09 \%$ \\
\hline S4 & 50 & $72,46 \%$ \\
\hline S5 & 0 & $0,00 \%$ \\
\hline
\end{tabular}

Ce patient est entré dans l'unité lors de la deuxième semaine, et a été transféré lors de la cinquième. Nous pouvons ici observer une saillance particulière dans la répartition des mots appartenant au champ lexical du traitement, correspondant à la difficulté éprouvée par les soignants à gérer les épisodes de crises de ce patient. Le point culminant de l'utilisation de ce vocabulaire étant à la quatrième semaine, soit juste avant que le patient ne soit transféré dans une Unité pour Malades Difficiles.

Ces différentes manipulations et explorations du corpus nous permettent donc de voir deux tendances dans les réunions de relèves infirmières. D'une part, le traitement - et par extension les discussions qui le concernent - est évolutif, selon l'état du patient. D'autre part, la prédominance, dans nos données, des tokens relatifs aux traitements est énoncée par des infirmiers, ce qui rend leur statut intrinsèquement lié à cette thématique.

\section{Conclusion}

Le corpus réalisé dans cette étude et son analyse nous permettent de répondre aux hypothèses de départ, bien qu'il existe des biais d'analyse dont un qui est lié à la taille de l'échantillon. Nous retenons deux enseignements.

Premièrement, les stratégies d'expressions orales et écrites témoignent d'abord des actions déjà réalisées. Toutefois cette proportion est majoritaire à l'écrit, probablement dans un objectif de traçabilité. Les actions à venir se retrouvent elles surtout à l'oral dans une volonté de transmettre les actions de soins encore à réaliser. Deuxièmement, cette comparaison permet de mettre en avant le rôle et la place des proches dans l'environnement immédiat des patients. De façon très majoritaire, ils sont en effet uniquement présents pendant les relèves orales.

Mais au-delà des comparaisons, nous pouvons souligner la complémentarité des relèves orales et écrites qui permettent au quotidien des transmissions d'informations au service du soin. Les interactions pluri professionnelles sur les temps de relèves favorisent la coconstruction des réflexions cliniques, il ne s'agit donc pas uniquement d'une transmission d'information (voir Colón De Carvajal, Maritaud \& Chalancon, 2020). La préservation des temps de relèves orales semble être un enjeu pour la qualité des soins, au vu de ces particularités son inscription dans les démarches qualités hospitalières témoignent d'ailleurs de l'importance de celle-ci. Le travail collaboratif réalisé entre les équipes de soins et l'équipes de recherche, bien que parfois difficile à mettre en place, nous montre qu'il est possible de tirer des enseignements contributifs à la qualité des soins et aux sciences du langage. 


\section{Références}

Abraham Joanna, Kannampallil Thomas, Brenner Corinne, Lopez Karen D., Almoosa Khalid F., Patel Bela et Patel Vimla L. (2016). Characterizing the structure and content of nurse handoffs: A Sequential Conversational Analysis approach. Journal of biomedical informatics, vol. 59, p. 76-88. https://doi.org/10.1016/j.jbi.2015. 11.009

Bangerter Adrian, Mayor Eric et Pekarek Doehler Simona. (2011). Reported speech in conversational storytelling during nursing shift handover meetings. Discourse Processes, vol. 48, n³, p. 183-214. https://doi.org/10.1080/0163853X.2010.519765

Cleary Michelle, Walter Garry and Horsfall Jan. (2009). Handover in psychiatric settings: is change needed?. Journal of psychosocial nursing and mental health services, vol. 47, n³, p. 28-33. https://doi.org/10.3928/02793695-20090301-02

Colón de Carvajal Isabel, Maritaud Louis, Chalancon Benoit et Lascar Justine. (2020). De la transmission d'informations cliniques au partage de savoir lors de relèves infirmières. TIPA. Travaux interdisciplinaires sur la parole et le langage, vol. 36. DOI : https://doi.org/10.4000/tipa.4032

Cowan Darrin, Brunero Scott, Luo Xiaoou, Bilton Dean and Lamont Scott. (2018). Developing a guideline for structured content and process in mental health nursing handover. International journal of mental health nursing, vol. $27, \mathrm{n}^{\circ} 1, \mathrm{p} .429-439$. https://doi.org/10.1111/inm.12337

Grosjean Michelle. (1997). Les relèves orales entre équipes infirmières à l'hôpital. Des structurations discursives aux pratiques cognitives et sociales. Pratiques sociales et médiations symboliques, p. 137-152.

Grosjean Michelle et Lacoste Michèle. (1999). Communication et intelligence collective. Paris cedex 14, France: Presses Universitaires de France. https://doi.org/10.3917/puf.grosj.1999.01

Grosjean Michelle. (2004). From multi-participant talk to genuine polylogue: shift-change briefing sessions at the hospital. Journal of Pragmatics, vol. 36, $\mathrm{n}^{\circ} 1$, p. 25-52. https://doi.org/10.1016/S0378-2166(03)00035-3

Groupe ICOR. (2006). La démarche ethnographique. Disponible sur CORINTE: http://icar.cnrs.fr/projets/corinte/recueil/demarche ethnographique.htm, consulté le 10 décembre 2021.

Lanza Danièle, Longchamp Philippe, Seferdjeli Laurence and Müller Ronald. (2004). Entre paroles et actions, appréhender la complexité de la coopération en milieu hospitalier. Cahiers de l'ILSL, vol. 16, p. 97-130.

LeBaron Curtis, Christianson Marlys K., Garrett Lyndon and Ilan Roy. (2016). Coordinating flexible performance during everyday work: An ethnomethodological study of handoff routines. Organization Science, vol. 27, n³, p. 514-534. https://doi.org/10.1287/orsc.2015.1043

Mori Junko, Imamura Akiko and Shima Chiharu. (2017). Epistemic management in the material world of workplace: A study of nursing shift handovers at a Japanese Geriatric Healthcare Facility. Journal of Pragmatics, vol. 109, p. 64-81. https://doi.org/10.1016/j.pragma.2017.01.002 